\title{
BASE SEQUENCE SPECIFICITY OF COUNTERION BINDING TO DNA: WHAT CAN MD SIMULATIONS TELL US?
}

\begin{tabular}{|r|l|}
\hline Journal: & Canadian Journal of Chemistry \\
\hline Manuscript ID & Cjc-2016-0296.R1 \\
\hline Manuscript Type: & Article \\
\hline Date Submitted by the Author: & 31-Aug-2016 \\
\hline Complete List of Authors: & $\begin{array}{l}\text { Atzori, Alessio; Università degli Studi di Cagliari, Dipartimento di Scienze } \\
\text { Chimiche e Geologiche } \\
\text { Liggi, Sonia; Università degli Studi di Cagliari, Dipartimento di Scienze } \\
\text { Chimiche e Geologiche } \\
\text { Laaksonen, Aatto; Universita degli Studi di Cagliari, Dipartimento di } \\
\text { Scienze Chimiche e Geologiche; Stockholm University, Division of Physical } \\
\text { Chemistry, Arrhenius Laboratory; Science for Life Laboratory } \\
\text { Porcu, Massimiliano; Università degli Studi di Cagliari, Dipartimento di } \\
\text { Scienze Chimiche e Geologiche; Stockholm University, Division of Physical } \\
\text { Chemistry, Arrhenius Laboratory } \\
\text { Lyubartsev, Alexander; Stockholm University, Division of Physical } \\
\text { Chemistry, Arrhenius Laboratory } \\
\text { Saba, Giuseppe; Università degli Studi di Cagliari, Dipartimento di Scienze } \\
\text { Chimiche e Geologiche } \\
\text { Mocci, Francesca; Università degli Studi di Cagliari, Dipartimento di Scienze } \\
\text { Chimiche e Geologiche; Stockholm University, Division of Physical } \\
\text { Chemistry, Arrhenius Laboratory }\end{array}$ \\
\hline Keyword: & \begin{tabular}{l} 
DNA, MD simulations, Solvation, Ion Interactions, sequence specificity \\
\hline
\end{tabular} \\
\hline
\end{tabular}


 BASE SEQUENCE SPECIFICITY OF COUNTERION BINDING TO} DNA: WHAT CAN MD SIMULATIONS TELL US?

3 Alessio Atzori, ${ }^{1}$ Sonia Liggi, ${ }^{1}$ Aatto Laaksonen, ${ }^{1,2,3}$ Massimiliano Porcu, ${ }^{1,2}$

4 Alexander P. Lyubartsev, ${ }^{2}$ Giuseppe Saba, ${ }^{1}$ and Francesca Mocci ${ }^{1,2,3}$

5

$6{ }^{1}$ Dipartimento di Scienze Chimiche e Geologiche, Università di Cagliari, Cittadella

7 Universitaria di Monserrato, I-09042 Monserrato (CA), Italy

$8{ }^{2}$ Division of Physical Chemistry, Arrhenius Laboratory, Stockholm University, S-10691

$9 \quad$ Stockholm, Sweden

$10 \quad{ }^{3}$ Science for Life Laboratory, 17121 Solna, Sweden

11

12 Corresponding author: Francesca Mocci, fmocci@unica.it, phone: 00393280110799, fax:

$13 \quad 00390706754388$ 
14 Abstract: Nucleic acids are highly charged biopolymers whose secondary structure is 15 strongly dependent on electrostatic interactions. Solvent molecules and ions are also believed to play an important role in mediating and directing both sequence recognition and 17 interactions with other molecules, such as proteins and a variety of ligands. Therefore, to 18 fully understand the biological functions of DNA, it is necessary to understand the 19 interactions with the surrounding counterions. It is well known that monovalent counterions can bind to the minor groove of DNA with consecutive sequences of four, or more, Adenine and Thymine (A-tracts) with relatively long residence times. However, much less is known about their binding to the backbone and to the major groove. In this work, we used molecular dynamics simulations to both investigate the interactions between the backbone and major groove of DNA and one of its physiological counterions $\left(\mathrm{Na}^{+}\right)$, and to evaluate the relationship between these interactions and the nucleotide sequence. Three dodecamers, namely CGAAAATTTTCG, CGCTCTAGAGCG, and CGCGAATTCGCG, were simulated using the Toukan-Rahman flexible SPC water model and Smith and Dang parameters for $\mathrm{Na}^{+}$, revealing a significant sequence-dependence on the ion binding to both backbone and major groove. In the absence of experimental data on the atomistic details of the studied interactions, the reliability of the results was evaluated performing the simulations with additional sets of potential parameters for ions and solvent, namely the Aqvist or the Joung and Cheatham ion parameters and the TIP3P water model. This allowed us to evaluate the results by verifying which features are preserved independently from the parameters adopted.

34 Keywords: DNA-counterion interactions, DNA sequence-specificity, Ion parameters, 35 molecular dynamics 
37 The highly negatively charged nature of DNA affects its structure, flexibility and functions, causing strong intra- and interstrand repulsions, which are offset by the presence of solvent and ions surrounding DNA in physiological conditions. ${ }^{1}$ Consequently, the properties of nucleic acids cannot be fully understood without considering their interactions with the 41 solvent molecules and counterions..$^{2-5}$ Ever since the first analysis of a small DNA fragment 42 using atomic resolution X-ray performed in $1973,{ }^{6}$ the structural and functional roles of counterions were greatly stressed. In the following decade, it has been proposed that the selectivity of alkaline ions for specific portions of DNA, rich in adenine and thymine, could explain the stabilization of the B-DNA conformation. ${ }^{7}$ Nonetheless, until mid-1990s, the important role of the interactions with monovalent counterions on the sequence-dependent properties have been neglected in most investigations focusing on the DNA structure. Indeed, these interactions were commonly believed to be "delocalized" and independent from the base sequence. ${ }^{8-9}$ The lack of studies on the sequence-specific effects of these interactions can be partially explained by difficulties connected with their observations. More specifically, Xray diffraction studies of nucleic acids cannot distinguish between the isoelectronic $\mathrm{Na}^{+}$ions

52 and water molecules, as well as due to their irregular coordination structure around DNA. ${ }^{10}$

53 Similarly, high resolution solution NMR techniques are not able to provide atomistic details

54 of the interactions of DNA with alkaline ions. This is due to both fast ion exchange in NMR

55 time scale and to the lack of modification of the detected DNA signals by such ions. ${ }^{10}$

56 Given these experimental limitations, the development of both computational resources and 57 molecular modeling techniques have boosted the theoretical studies of nucleic acids and their 58 binding with physiological ions. ${ }^{11} \mathrm{~A}$ vital interest to explain the role of monovalent 59 counterions on DNA sequence specific features ${ }^{11-13}$ originated from the molecular dynamics 60 (MD) investigations performed by Young et al. . ${ }^{14-15}$ In particular, their investigation showed 
61 how counterions interact preferentially with the minor groove of consecutive sequences of AT

62 base pairs without any 5'-TpA-3' steps (defined as A-tracts). Following these results, several

63 experimental studies were performed ${ }^{15-18}$ provoking a significant debate especially among

64 crystallographers. $^{12-13,19-21}$

65 Most of the recent MD studies on sequence specificity ${ }^{12,22-27}$ have been focusing on the

66 interactions of counterions with the minor groove, while those with the backbone and major

67 groove regions have received less consideration. In order to bridge this gap, the relationships

68 between the DNA sequence and the interactions of ions with the major groove and the

69 backbone have been evaluated in this study. To this purpose, three different sequences were

$70 \quad$ simulated (See Figure 1):

71 - CGAAAATTTTCG (from now on called A4T4), containing an 8 bases long A-tract;

72 - CGCGAATTCGCG (known as Drew Dickerson Dodecamer and hence called DDD ${ }^{28}$ ), with

73 a 4 bases long A-tract;

74 - CGCTCTAGAGCG (from now on called TA), having the same nucleotide composition of

75 DDD but different order of the bases, and hence lacking an A-tract.

76 To partly compensate for the lack of experimental data on the atomistic details of the studied

77 interactions, the results of the simulations were cross-checked by performing the simulations

78 using three combinations of water model (TIP3P $\mathrm{P}^{29}$ and flexible $\mathrm{SPC}^{30}$ ) and ion parameters

79 (Smith and Dang, ${ }^{31}$ Joung and Cheatham, ${ }^{32}$ and $\AA$ Avist ${ }^{33}$ ), as detailed in the computational

80 methods section. The used combinations of potential parameters were chosen due to their

81 wide use in the previous investigations aimed to study the interactions of B-DNA with

82 counterions (see the recent review by Mocci and Laaksonen, Table $1^{11}$ ), and to compare them

83 with a recently developed potential model ${ }^{32}$ to verify its performance on this type of

84 investigation. 
85 Therefore, while obtaining a representative and comprehensive picture of the ion /DNA 86 interactions using MD, we also tried to establish a well-defined protocol using different 87 parameters to study interactions between counterions and specific DNA sequences, as we did 88 recently in a study on DNA quadruplexes. ${ }^{34}$

\section{Computational methods}

90 The initial structures of the three duplexes were built using the Nucgen module of the 91 AMBER package. ${ }^{35}$ The experimental data for TA and DDD suggests that they are in the B 92 form. ${ }^{28}$ Even if no crystallographic structures are available for A4T4, it was generated in a 93 canonical B-DNA form, similarly to the other two oligomers. Each duplex was immersed in a 94 truncated octahedral box containing 4500 water molecules and $22 \mathrm{Na}^{+}$counterions. Three

95 different simulations were performed for each sequence using the parmbsc 0 force field ${ }^{36}$ for 96 the DNA and three different combinations of solvent models and ions parameters:

97 Set 1: a refined model of water, the Toukan-Rahman flexible SPC model, ${ }^{30}$ with the Smith and Dang parameters for $\mathrm{Na}^{+} ; 31$

99 Set 2: $\mathrm{Na}^{+}$parameters recently developed by Joung and Cheatham ${ }^{32}$ along with TIP3P water 100 model; ${ }^{29}$

101 Set 3: the most widely used combination used in studies of B-DNA interaction with 102 counterions: Åqvist parameters ${ }^{33}$ for $\mathrm{Na}^{+}$together with the TIP3P water model. ${ }^{29}$

103 Numerical values of the used parameters are reported in Table 1.

105 Twenty-two $\mathrm{Na}^{+}$counterions were placed using the utility Xleap of the AMBER14 package ${ }^{35}$ 106 at the points of lowest Coulombic potential, which were calculated using a distance- 
107 dependent dielectric constant on a grid of $1 \AA$ resolution. 4500 water molecules were added 108 to fill a truncated octahedral box of side length $\sim 65 \AA$. The system was equilibrated with an 109 initial steepest descent minimization, followed by a 3 ps dynamics with positional constraints 110 on the $\mathrm{Na}^{+}$and DNA atoms; the constraints were then gradually removed by several 111 minimization runs, those on $\mathrm{Na}^{+}$being relaxed faster. The system was then heated to $300 \mathrm{~K}$ 112 over 2 ps and followed by 400 ns of dynamics using Mdynamix ${ }^{37}$ in NPT ensemble at $300 \mathrm{~K}$ 113 and 1 bar (using Nose-Hoover algorithm with time constants of 30.0 fs for thermal 114 fluctuations and 700.0 fs for volume fluctuations). The double time step algorithm of 115 Tuckerman was used, with a time step of 0.2 fs for short (cut off=5 $\AA$ ) intra and inter116 molecular interactions, and a time step of 2 fs for the others (cut off $=14 \AA$ ). Electrostatic 117 interactions were calculated using the particle mesh Ewald method. ${ }^{38}$ The cutoff radius in the 118 real space (Rcut) was set to $14 \AA$, while the cutoff radius in the reciprocal space, $k$ max, was 119 determined from the condition $\exp \left[-\alpha^{2} k \max ^{2} /[R \operatorname{cut} \alpha]^{2}\right]=\exp [-8$.$] ; the screening parameter \alpha$ 120 was taken as $\alpha$ cut $=2.7$.

121 System preparation for set 2 and 3

122 System setup and equilibration are the same as the ones described for set 1 . Four-hundred ns 123 of dynamics were carried out using the PMEMD module of AMBER $14^{35}$ with a time step of 1242 fs and SHAKE constraints on hydrogens (tolerance $=0.00005$ ). The Berendsen algorithm ${ }^{39}$ 125 with a coupling constant of 3 ps was utilized to maintain constant temperature $(300 \mathrm{~K})$ and 126 pressure (1 bar). In order to reduce the computational cost of the performed calculations, we 127 evaluated the effect of the reduction of the cutoff radius for non-bonded interactions from 14 $128 \AA$ (as in set 1) to $9 \AA$. This reduction did not lead to any significant variation in the 129 interactions trend of DNA with $\mathrm{Na}^{+}$counterions (Supplementary Information Figure 1). 130 Electrostatic interactions were calculated by the particle mesh Ewald method as implemented 131 in AMBER $14,{ }^{35}$ with a cubic B-spline interpolation order and 0.00001 tolerance for the 
132 direct space sum cutoff. Periodical removal of the net center-of-mass momentum was applied 133 in order to eliminate the "flying ice cube" phenomenon. ${ }^{40}$ Volume, pressure, density, and 134 temperature all reached their stationary values within $15 \mathrm{ps}$.

135 Analysis of the results

136 Normalized radial distribution functions (RDFs) between the $\mathrm{Na}^{+}$ions and selected reference 137 atoms of DNA in the backbone and in the major groove (high-lighted in Figure 2) and 138 residence times of $\mathrm{Na}^{+}$to the oxygens of the phosphate groups of each sequence were 139 calculated using an in-house version of the Tranal module of Mdynamix. ${ }^{37}$ In the calculation 140 of the RDFs the two terminal base pairs of each duplex were neglected, considering only the 141 eight central base pairs. The residence time of direct binding events of counterions with DNA 142 were calculated for each filament in the $5^{\prime} \rightarrow 3^{\prime}$ direction. The binding events were estimated 143 as a weighted average of the contact residence times being equal or longer than 3 ps. Contacts 144 are considered to be interrupted if the ion moves away from the reference electronegative 145 atom for more than 4 ps and $3.2 \AA$. $\mathrm{VMD}^{41}$ was used to visualize trajectories and to generate 146 graphical representations of each system.

\section{$147 \quad \underline{\text { Results and discussion }}$}

148 During the simulations different types of interactions between counterions and DNA can 149 occur, such as direct or solvent mediated contacts. In order to quantify and classify these 150 interactions, we performed a set of analyses providing complementary information. In the 151 following paragraphs, we first discuss the results obtained employing the set 1 parameters for 152 the three oligomers analyzing the sequence dependence of the interactions in the backbone 153 and in the major groove using RDFs. Thereafter, we compare the results obtained with set 1 154 with those from set 2 and 3 parameters using both RDFs and weighted average residence 155 times. 
156 Are $\mathrm{Na}^{+}$interactions with the backbone and major groove of DNA sequence-dependent?

157 The two negatively charged oxygen atoms of the phosphate groups of DNA, namely O1P and $158 \mathrm{O} 2 \mathrm{P}$, possess an opposite spatial orientation: $\mathrm{O} 1 \mathrm{P}$ faces the minor groove, while $\mathrm{O} 2 \mathrm{P}$ is 159 directed toward the major groove (Figure 3a). Consequently, their interactions with the 160 counterions are expected to be different.

161 The calculated RDFs show that, independently from the sequence, the direct contact between $162 \mathrm{Na}^{+}$and the phosphate oxygen atoms (peak at $\sim 2.5 \AA$ ) occurs preferentially with O1P 163 (Figure 3b, black line), confirming what has been observed in earlier investigations. ${ }^{26,42} \mathrm{~A}$ 164 sequence specific feature can be observed by comparing the intensity of the first RDF peak 165 among the three sequences: the probability of binding to O1P increases with the length of the 166 A-tract. Indeed, the first peak intensity is highest for the A4T4 oligomer, followed by that for 167 DDD and the lowest being that for the TA (See Table S1 in the supplementary information 168 for the numeric values of the maxima for each RDF peak). On the contrary, this trend is not 169 observed for the binding of $\mathrm{Na}^{+}$to $\mathrm{O} 2 \mathrm{P}$.

170 Concerning the major groove, the RDFs of $\mathrm{Na}^{+}$with respect to the selected electronegative 171 atoms (Figure 4a) are shown in Figure 4b; in Table S2 of the supplementary information are 172 listed the numeric values of the maxima for each RDF peak. The comparison of the RDFs of $173 \mathrm{Na}^{+}$indicates that the probability of $\mathrm{Na}^{+}$to bind electronegative atoms of DNA is dependent 174 on the base pair sequences. For instance, the direct binding to $\mathrm{T}_{\mathrm{O} 4}$ is a rare event in the major 175 groove of A-tracts (absence of a green peak at $2.5 \AA$ for A4T4). On the contrary, when a 176 thymine is not embedded in an A-tract sequence, the probability of a direct binding is 177 moderately high (green peaks at $2.5 \AA$ for TA and DDD).

178 By comparing the intensity of the peaks corresponding to the direct binding to any of the 179 atoms in the major groove of TA and DDD, it can be observed that the intensities are always 
180 higher for the direct contact with the former. On the overall, in contrast with what has been 181 observed for the interactions of $\mathrm{Na}^{+}$with backbone atoms, the presence of an A-tract is associated with a lower probability of finding an ion directly bound to the major groove.

183 Noteworthy, the peaks with the highest intensity for each oligomer belong to $A_{\mathrm{N} 7}$ 184 independently from the sequence considered. When the guanine base is present in the sequence, the binding to $G_{N 7}$ is less probable than to $G_{06}$. Direct contacts between counterions and $\mathrm{C}_{\mathrm{N} 4}$ and $\mathrm{A}_{\mathrm{N} 6}$ are negligible: in fact, the presence of partial positive charges on the nitrogen of these atoms causes an electrostatic repulsion of the counterions.

In order to interpret the peaks at distances greater than $2.5 \AA$, further analyses are required.

Generally, the second peak can indicate a water mediated contact ( $5 \AA$, Figure 5a), or an interaction with an electronegative atom located in a complementary or nearby base (from $\sim 4$ to $\sim 6 \AA$ Figure $5 \mathrm{~b}$ ). An example of the latter is the direct contact of $\mathrm{Na}^{+}$with $\mathrm{A}_{\mathrm{N} 7}$, which is reflected also on the peaks of $\mathrm{A}_{\mathrm{N} 6}$ (yellow peaks around $\sim 4 \AA$ ) and $\mathrm{T}_{\mathrm{O} 4}$ (green peaks around $\sim 6 \AA$ ) in Figure $4 \mathrm{~b}$. The third peak may originate from a direct contact of the ion in the opposite groove. In particular, the peaks at $\sim 7 \AA$ for $\mathrm{T}_{\mathrm{O} 4}$ and $\sim 8 \AA$ for $\mathrm{A}_{\mathrm{N} 7}$ in DDD are caused by a $\mathrm{Na}+$ interacting with $\mathrm{T}_{\mathrm{O} 2}$ located in the minor groove (Figure $5 \mathrm{c}$ ). Indeed, the RDFs calculated for $\mathrm{T}_{\mathrm{O} 2}$ (as shown in SI Figure 2) indicate that the intensity of its first peak is correlated to those observed in the third peaks of the major groove.

\section{Comparison of the results obtained with different ion parameters and solvent models}

199 In order to evaluate the impact of different parameters for sodium and water on the interactions between DNA and counterions, we compared the results obtained with the three different combinations of parameters described in the computational methods section. 
202 The RDFs represented in Figure 6 indicate how the affinity of $\mathrm{Na}^{+}$for the oxygen atoms of

203 the backbone is highly dependent on the set of parameters employed, with set 3 leading to a

204 remarkable lower affinity than the other two sets. This result is in agreement with what was

205 observed by Noy et al. ${ }^{43}$ when comparing Åqvist with Joung \& Cheatham parameters for $\mathrm{Na}^{+}$.

206 Even if the binding affinity varies with the set, the main sequence specific features of the ion

207 binding are observed with all sets. Namely, all sets show a preferential binding of the

208 counterions to $\mathrm{O} 1 \mathrm{P}$ compared to $\mathrm{O} 2 \mathrm{P}$. Further, this preference increases when A-tracts are

209 present, and with the length of the A-tract. To better quantify this trend, in Table S1 of the

210 supplementary information we report the heights of the peaks, and the ratio between the

211 peaks maxima of the O1P and O2P RDFs. It can be seen that the mentioned ratio decreases

212 with the length of the A-tract, with the following trend within all the combination of

213 parameters: A4T4 > DDD > TA.

214 We verified whether the higher probability of binding to the phosphate of A-tract containing 215 sequences is correlated to a longer residence time in the binding sites. In Figure 7 are plotted 216 the weighted average residence times of the direct binding events occurring along the 217 backbone. For more details on this analysis, please refer to the computational methods 218 section. It is useful to recall that the two filaments of each oligomer in this study have the 219 same base sequence; therefore, the same trend in the residence time can be expected. More 220 precisely, we expect the residence time along the two chains to overlap when full 221 convergence is reached. This is observed for A4T4 and DDD with all sets of parameters. For 222 these sequences, residence times of $\mathrm{Na}^{+}$are higher when the ion binds to $\mathrm{O} 1 \mathrm{P}$ atoms rather 223 than $\mathrm{O} 2 \mathrm{P}$ atoms, especially in A-tracts. In addition, the residence time increases along the A224 tract in the $5^{\prime} \rightarrow 3^{\prime}$ direction. However, this trend is observed only with two of the three sets 225 analyzed: the widely used Åqvist parameters (set 3) does not reveal this sequence specific 
226 feature; in addition, the residence times of binding events are generally shorter $(\sim 100 \mathrm{ps})$

227 than those observed with the other two sets. On the other hand, no clear difference in 228 residence times between $\mathrm{O} 1 \mathrm{P}$ and $\mathrm{O} 2 \mathrm{P}$ is observed for the TA sequence with any of the sets 229 of parameters.

230 On the basis of these analyses, we can conclude that according to the simulations performed 231 with set 1 and set 2, both the probability of the direct binding event and its residence time are 232 highly affected by the sequence. This does not apply to the simulations performed with set 3 , 233 where the sequence-specificity in residence times is not clearly observed.

234 Concerning the major groove, the comparison of the RDFs calculated from the simulations 235 with the three different sets of parameters indicates that all the combinations of potential 236 models provide the same qualitative information concerning the sequence specificity of the 237 ion binding (Figure 8). In fact, all of the observations made in the previous section 238 concerning the sequence specificity resulting from the simulations with set 1 , are still valid 239 with sets 2 and 3 and will not be further commented here. Independently from the sequence 240 and the set of parameters used, $\mathrm{A}_{\mathrm{N} 7}$ is the most favorable binding site of sodium ion, followed 241 by $\mathrm{G}_{\mathrm{O} 6}$ for DDD and TA.

242 However, there are some differences that deserve attention: with set 3 , the binding to $A_{\mathrm{N} 7}$ in 243 both TA and DDD is highly favored compared to that to other electronegative atoms in the 244 groove, while, this preference is less pronounced with the other two sets of parameters. 245 Another difference concerns the peak at $\sim 7 \AA$ for $\mathrm{T}_{\mathrm{O} 4}$, which is clearly observed in set 1 , 246 especially for DDD, and is less evident in the simulations with set 2 and 3 . This is due to the 247 diverse probability of $\mathrm{Na}+$ in binding to $\mathrm{T}_{\mathrm{O} 2}$ in the minor groove. As shown in the supporting 248 information (Figure S2), the intensity of the peak for the direct contact between $\mathrm{Na}^{+}$and $\mathrm{T}_{\mathrm{O} 2}$ 249 of DDD is noticeably higher for set 1 than for the other two sets. 
250 Residence time of direct binding events of $\mathrm{Na}^{+}$to the electronegative atoms of the major 251 groove for the two filaments of each duplex are shown in Figure 9. As mentioned previously, 252 we expect an overlap between the two filaments when a full convergence is achieved. This is 253 observed with most of the simulations, with only few irregularities observed. This is 254 somehow unexpected considered that the degree of convergence for the interactions in the 255 major groove is higher than the one observed for the backbone atoms.

256 As observed previously for the binding to the backbone, set 3 parameters leads to short 257 residence times of binding events $(\sim 100 \mathrm{ps})$, while set 1 produces the highest residence times 258 (between 300 and $1200 \mathrm{ps).}$

259 The residence times exhibit slight sequence specificity, which is possible to observe within all 260 sets of parameters. In detail, the residence time of the binding to the thymine bases in the 261 center of A-tracts is not only a rare event, as indicated by the RDFs, but is also characterized 262 by a short residence time. Longer residence time for the binding to thymine is observed when 263 the base is located at the end of the A-tract, or when is not part of an A-tract. On the contrary, 264 the residence time of $\mathrm{Na}^{+}$binding to adenine is slightly higher in the center of the A-tract.

Differently from what has been observed for the interactions with the oxygen atoms of the 266 phosphate, an end effect is observed in the residence time of the binding of the ion to 267 guanine: in fact, the residence time of terminal guanines are shorter than those observed for 268 the same base in the center of the oligomer. However, it cannot be excluded that this result is 269 also due to the lack of adjacent purine type bases.

270 As expected from the RDFs, where the first peak for the binding to cytosine is absent or very 271 low indicating an extremely rare event (Figure 8), residence times are noticeably short, or 272 even equal to zero, since no binding event is observed. 


\section{Conclusions}

274 MD simulations of three different DNA sequences allowed us to verify that the interactions of 275 DNA with $\mathrm{Na}^{+}$counterions are highly sequence dependent not only in the minor groove, as 276 shown in previous studies, but also in the backbone and in the major groove. In fact, the 277 probability of the ions to interact with a given base and its close surrounding varies 278 noticeably between the A-tract and non A-tract sequences. The three combinations of solvent 279 and counterions parameters agreed mutually on several results:

280 (i) $\mathrm{Na}^{+} / \mathrm{DNA}$ direct contact is less probable in the major groove of oligomers containing A281 tract;

(ii) Independently from the sequence, $A_{\mathrm{N} 7}$ is the most favorite site in the major groove for the interactions of $\mathrm{Na}^{+}$;

284 (iii) The binding of the counterions with the outermost atoms of the duplex DNA, i.e. the oxygen atoms of the phosphates, are also affected by the sequence, with the A-tracts having higher probability of direct binding with $\mathrm{Na}^{+}$.

287 However, the details of the sequence-specific features of the interactions should be judged carefully, since they might vary with the models employed for the solvent and counterions. Indeed, the different sets adopted in this study do not give a unified picture of the sequence dependence of the average residence time of the binding events occurring in the backbone. More specifically, the most widely used set of parameters used in the previous MD simulations of B-DNA interaction with $\mathrm{Na}+$, consisting of the combination of the amber adapted Åqvist parameters for the ion with TIP3P solvent model, differs noticeably from the

294 other sets. The differences observed are both quantitative and qualitative: set 3 leads to 
295 considerably lower residence times, and does not display the sequence dependence of the

296 residence times of the ion binding to the backbone.

\section{Acknowledgements}

298 F.M. gratefully acknowledges financial support from the Regione Autonoma della Sardegna, 299 through the Legge Regionale 07/09/2007 (code CRP-59740) and Prof. Giuseppe Saba for 300 technical support. A.L. wishes to acknowledge the Visiting Professor Programme financed 301 by the Regione Autonoma Sardegna and the Swedish Research Council (VR) for financial 302 support. All authors acknowledge the Swedish National Infrastructure for Computing (SNIC). 
303

304

305

306

307

308

309

310

311

312

313

314

315

316

317

318

319

320

321

322

323

324

325

326

327

\section{References}

1. Leslie, A. G.; Arnott, S.; Chandrasekaran, R.; Ratliff, R. L., J. Mol. Biol. 1980, 143 (1), 49 .

2. Jayaram, B.; Beyeridge, D. L., Annu. Rev. Biophys. Biomol. Struct. 1996, 25, 367. doi: 10.1146/annurev.bb.25.060196.002055.

3. Manning, G. S., Biophys. J. 2006, 90 (9), 3208. doi: 10.1529/biophysj.105.078865.

4. Subirana, J. A.; Soler-Lopez, M., Annu. Rev. Biophys. Biomol. Struct 2003, 32, 27. doi: 10.1146/annurev.biophys.32.110601.141726.

5. Auffinger, P.; Hashem, Y., Curr. Opin. Struct. Biol. 2007, 17 (3), 325. doi: 10.1016/j.sbi.2007.05.008.

6. Rosenberg, J. M.; Seeman, N. C.; Kim, J. J.; Suddath, F. L.; Nicholas, H. B.; Rich, A., Nature 1973, 243 (5403), 150.

7. Bartenev, V. N.; Golovamov Eu, I.; Kapitonova, K. A.; Mokulskii, M. A.; Volkova, L. I.; Skuratovskii, I., J. Mol. Biol. 1983, 169 (1), 217.

8. Deng, H.; Braunlin, W. H., J. Mol. Biol. 1996, 255 (3), 476. doi: 10.1006/jmbi.1996.0039.

9. Mocci, F.; Laaksonen, A. In Nuclear Magnetic Resonance: Volume 44, Vol. 44The Royal Society of Chemistry2015; pp 592.

10. Auffinger, P.; Grover, N.; Westhof, E., Met. Ions Life Sci. 2011, 9, 1.

11. Mocci, F.; Laaksonen, A., Soft Matter 2012, 8 (36), 9268. doi: 10.1039/C2SM25690H.

12. Hamelberg, D.; McFail-Isom, L.; Williams, L. D.; Wilson, W. D., J. Am. Chem. Soc. 2000, 122 (43), 10513. doi: 10.1021/ja0007071.

13. McConnell, K. J.; Beveridge, D. L., J. Mol. Biol. 2000, 304 (5), 803. doi: 10.1006/jmbi.2000.4167. 
328 14. Young, M. A.; Ravishanker, G.; Beveridge, D. L., Biophys. J. 1997, 73 (5), 2313. doi: $329 \quad 10.1016 / \mathrm{S} 0006-3495(97) 78263-8$.

330 15. Young, M. A.; Beveridge, D. L., J. Mol. Biol. 1998, 281 (4), 675. doi: $331 \quad 10.1006 /$ jmbi.1998.1962.

332 16. Denisov, V. P.; Halle, B., Proc. Natl. Acad. Sci. U.S.A. 2000, 97 (2), 629.

333 17. Hud, N. V.; Polak, M., Curr. Opin. Struct. Biol. 2001, 11 (3), 293.

334 18. Engelhart, A. E.; Morton, T. H.; Hud, N. V., Chem. Commun. (Camb.) 2009, (6), 647. 335 doi: 10.1039/b818409g.

336 19. Woods, K. K.; Maehigashi, T.; Howerton, S. B.; Sines, C. C.; Tannenbaum, S.; 337 Williams, L. D., J. Am. Chem. Soc. 2004, 126 (47), 15330. doi: 10.1021/ja045207x.

338 20. McFail-Isom, L.; Sines, C. C.; Williams, L. D., Curr. Opin. Struct. Biol. 1999, 9 (3), 339 298. doi: http://dx.doi.org/10.1016/S0959-440X(99)80040-2.

340 21. Woods, K. K.; McFail-Isom, L.; Sines, C. C.; Howerton, S. B.; Stephens, R. K.; 341 Williams, L. D., ., J. Am. Chem. Soc. 2000, 122 (7), 1546. doi: 10.1021/ja9919579.

342 22. Lyubartsev, A. P.; Laaksonen, A., J. Biomol. Struct. Dyn. 1998, 16 (3), 579. doi: $343 \quad 10.1080 / 07391102.1998 .10508271$.

344 23. Feig, M.; Pettitt, B. M., Biopolymers 1998, 48 (4), 199. doi: 10.1002/(SICI)1097345 0282(1998)48:4<199::AID-BIP2>3.0.CO;2-5.

346 24. Auffinger, P.; Westhof, E., J. Mol. Biol. 2000, 300 (5), 1113. doi: $347 \quad 10.1006 /$ jmbi.2000.3894.

348 25. Mocci, F.; Saba, G., Biopolymers 2003, 68 (4), 471. doi: 10.1002/bip.10334.

349 26. Mocci, F.; Laaksonen, A.; Lyubartsev, A.; Saba, G., J. Phys. Chem. B 2004, 108 (41), 350 16295. doi: 10.1021/jp047744+.

351 27. Ponomarev, S. Y.; Thayer, K. M.; Beveridge, D. L., Proc. Natl. Acad. Sci. U.S.A. 352 2004, 101 (41), 14771. doi: 10.1073/pnas.0406435101. 
353 28. Tereshko, V.; Urpi, L.; Malinina, L.; Huynh-Dinh, T.; Subirana, J. A., Biochemistry 354 1996, 35 (36), 11589. doi: 10.1021/bi9610481.

355 29. Jorgensen, W. L.; Chandrasekhar, J.; Madura, J. D.; Impey, R. W.; Klein, M. L., J. 356 Chem. Phys. 1983, 79 (2), 926. doi: doi:http://dx.doi.org/10.1063/1.445869.

357 30. Toukan, K.; Rahman, A., Phys. Rev. B 1985, 31 (5), 2643.

358 31. Smith, D. E.; Dang, L. X., J. Chem. Phys. 1994, 100 (5), 3757. doi: 359 doi:http://dx.doi.org/10.1063/1.466363.

360 32. Joung, I. S.; Cheatham, T. E., 3rd, J. Phys. Chem. B 2008, 112 (30), 9020. doi: $361 \quad 10.1021 / j p 8001614$.

362 33. Ȧqvist, J., J. Phys. Chem. 1990, 94 (21), 8021. doi: 10.1021/j100384a009.

363 34. Rebic, M.; Laaksonen, A.; Sponer, J.; Ulicny, J.; Mocci, F., J. Phys. Chem. B 2016, $364120(30), 7380$. doi: 10.1021/acs.jpcb.6b06485.

365 35. D.A. Case, V. B., J.T. Berryman, R.M. Betz, Q. Cai, D.S. Cerutti, T.E. Cheatham, III, 366 T.A. Darden, R.E.; Duke, H. G., A.W. Goetz, S. Gusarov, N. Homeyer, P. Janowski, J. Kaus, 367 I. Kolossváry, A. Kovalenko,; T.S. Lee, S. L., T. Luchko, R. Luo, B. Madej, K.M. Merz, F. 368 Paesani, D.R. Roe, A. Roitberg, C. Sagui,; R. Salomon-Ferrer, G. S., C.L. Simmerling, W. 369 Smith, J. Swails, R.C. Walker, J. Wang, R.M. Wolf, X.; Kollman, W. a. P. A. AMBER 14: 370 University of California, San Francisco, 2014.

371 36. Perez, A.; Marchan, I.; Svozil, D.; Sponer, J.; Cheatham, T. E., 3rd; Laughton, C. A.; 372 Orozco, M., Biophys. J. 2007, 92 (11), 3817. doi: 10.1529/biophysj.106.097782.

373 37. Lyubartsev, A. P.; Laaksonen, A., Comput. Phys. Commun. 2000, 128 (3), 565. doi: 374 http://dx.doi.org/10.1016/S0010-4655(99)00529-9.

375 38. Darden, T.; York, D.; Pedersen, L., J. Chem. Phys. 1993, 98 (12), 10089. doi: $376 \quad 10.1063 / 1.464397$. 
377 39. van Gunsteren, W. F.; Berendsen, H. J.; Hermans, J.; Hol, W. G.; Postma, J. P., Proc. 378 Natl. Acad. Sci. U.S.A. 1983, 80 (14), 4315.

379 40. Harvey, S. C.; Tan, R. K. Z.; Cheatham, T. E., J. Comput. Chem. 1998, 19 (7), 726. 380 doi: 10.1002/bip.10334.

381 41. Humphrey, W.; Dalke, A.; Schulten, K., J. Mol. Graph. 1996, 14 (1), 33. doi: 382 http://dx.doi.org/10.1016/0263-7855(96)00018-5.

383 42. Korolev, N.; Lyubartsev, A. P.; Laaksonen, A.; Nordenskiold, L., Nucleic Acids Res. 384 2003, $31(20), 5971$.

385 43. Noy, A.; Soteras, I.; Luque, F. J.; Orozco, M., Phys. Chem. Chem. Phys. 2009, 11 (45), 386 10596. doi: 10.1039/b912067j. 
$\underline{\text { Tables }}$

389 Table 1- Ion potential parameters used in the MD Simulations presented in this study. Lennard-Jones parameters

\begin{tabular}{ccccc} 
Set name & CIp $^{\mathrm{a}}$ & $\mathrm{W}^{\mathrm{b}}$ & $\sigma(\mathrm{nm})$ & $\varepsilon(\mathrm{kJ} / \mathrm{mol})$ \\
\cline { 3 - 4 } & & & & \\
\hline set 1 & SD & SPC & $2.350 \times 10^{-1}$ & $5.440 \times 10^{-1}$ \\
set 2 & JC & TIP3P & $2.439 \times 10^{-1}$ & $3.658 \times 10^{-1}$ \\
set 3 & AA & TIP3P & $3.328 \times 10^{-1}$ & $1.159 \times 10^{-2}$ \\
& & & & \\
\hline
\end{tabular}

$390 \quad{ }^{a}$ Counterion parameters with the following abbreviations: SD: Smith and Dang; ${ }^{31}$ JC: Joung 391 and Cheatham; ${ }^{32}$ AA: Amber-adapted Aqvist parameters. ${ }^{33}{ }^{b}$ Water model: flexible SPC 392 model; ${ }^{30}$ TIP3P model. ${ }^{29}$ 
Fig. 1 - The three DNA sequences considered in this study. Backbone atoms are in ribbon and the atoms of the bases in licorice representations, respectively. The bases are color-coded as shown in the legend. Sodium ions are represented as spheres in cyan.

Fig. $2-\mathrm{A} \cdot \mathrm{T}$ and $\mathrm{C} \cdot \mathrm{G}$ in the canonical Watson-Crick DNA base pairing; electronegative reference atoms used in the analysis of ion binding in the major groove and in the backbone are labeled.

Fig. 3 - (a) The DDD sequence with highlighted oxygen atoms of the phosphates O1P (black spheres) and O2P (red spheres). (b) RDFs of $\mathrm{Na}^{+}$with respect to O1P (black lines) and O2P (red lines) for the three oligomers A4T4, TA and DDD obtained with set 1 parameters.

Fig. 4 - (a) The DDD sequence with the highlighted reference atoms of the major groove. (b) RDFs of $\mathrm{Na}^{+}$with respect to the reference atoms of the major groove for the three oligomers A4T4, TA and DDD obtained with set 1 parameters. Color codes for each atom are shown in 407 the legend.

Fig. 5 - a) Representative conformation of a water-mediated contact of a sodium ion with $\mathrm{A}_{\mathrm{N} 7}$ to explain the peak of the RDF at $\sim 4.5 \AA \AA$. b) Representative conformation of a direct contact of a sodium ion with $\mathrm{A}_{\mathrm{N} 7}$ to explain the peaks at $\sim 4.5 \AA$ and $\sim 6 \AA$ observed in the 411 peaks of RDF respectively for $\mathrm{A}_{\mathrm{N} 6}$ and $\mathrm{T}_{\mathrm{O} 4}$. c) Representative conformation of a direct contact 412 of a sodium ion with $\mathrm{T}_{\mathrm{O} 2}$ to explain the peaks at $\sim 6 \AA$ observed in $\mathrm{RDF}$ for $\mathrm{T}_{\mathrm{O} 4}$.

413 Fig. 6 - RDFs of $\mathrm{Na}^{+}$with respect to O1P (black) and O2P (red) for the three oligomers 414 A4T4, TA and DDD obtained respectively with set 1, 2 and 3 parameters.

415 Fig. 7 - Weighted average residence time of $\mathrm{Na}^{+}$in direct contact with the oxygen atoms of 416 the phosphate groups for the three oligomers A4T4, TA and DDD obtained respectively with 
417 set 1, 2 and 3 parameters. both filaments are represented in the 5 ' $\rightarrow 3^{\prime}$ direction. Color code:

418 black, continuous line $=\mathrm{O} 1 \mathrm{P}$ filament 1 ; black dashed line $=\mathrm{O} 1 \mathrm{P}$ filament 2; red, continuous

419 line $=\mathrm{O} 2 \mathrm{P}$ filament $1 ;$ red dashed line $=\mathrm{O} 2 \mathrm{P}$ filament 2.

420 Fig. 8 - RDFs of $\mathrm{Na}^{+}$with respect to reference atoms of the major groove for the three

421 oligomers A4T4, TA and DDD obtained respectively with set 1, 2 and 3 parameters. Color

422 codes for each electronegative atom are shown in the legend.

423 Fig. 9 - Weighted average residence time of $\mathrm{Na}^{+}$in direct contact with electronegative atoms 424 of the major groove for the three oligomers A4T4, TA and DDD obtained respectively with 425 set 1,2 and 3 parameters. Colour code: black line $=$ filament 1 ; red line $=$ filament 2. 


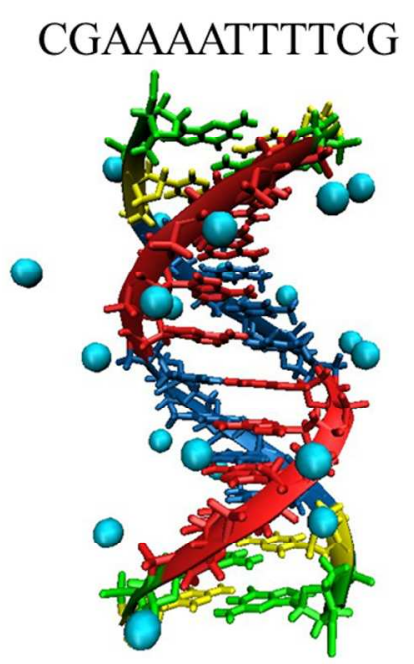

A4T4

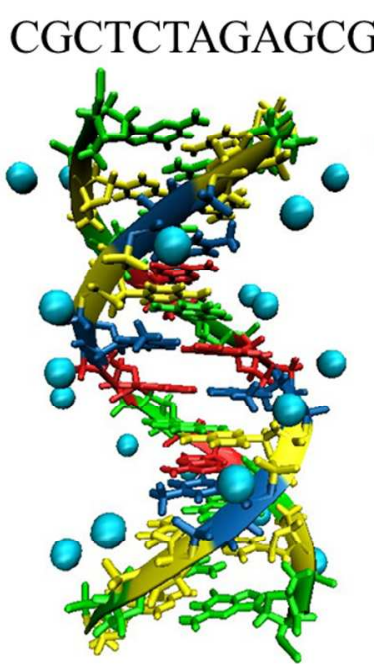

TA

\section{CGCGAATTCGCG}

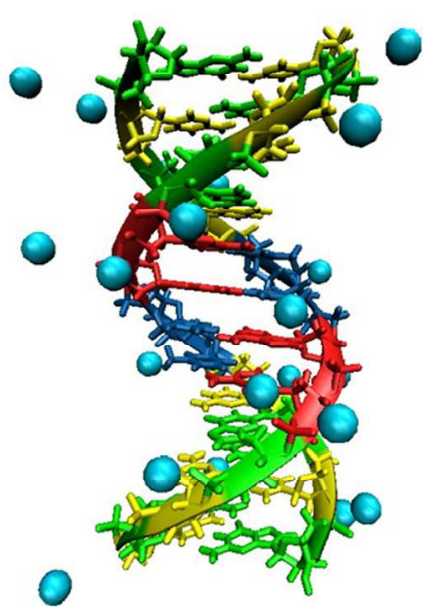

DDD

Adenine - Thymine Guanine $=$ Cytosine

Fig. 1 - The three DNA sequences considered in this study. Backbone atoms are in ribbon and the atoms of the bases in licorice representations, respectively. The bases are color-coded as shown in the legend. Sodium ions are represented as spheres in cyan.

Figure 1

$171 \times 106 \mathrm{~mm}(300 \times 300 \mathrm{DPI})$ 

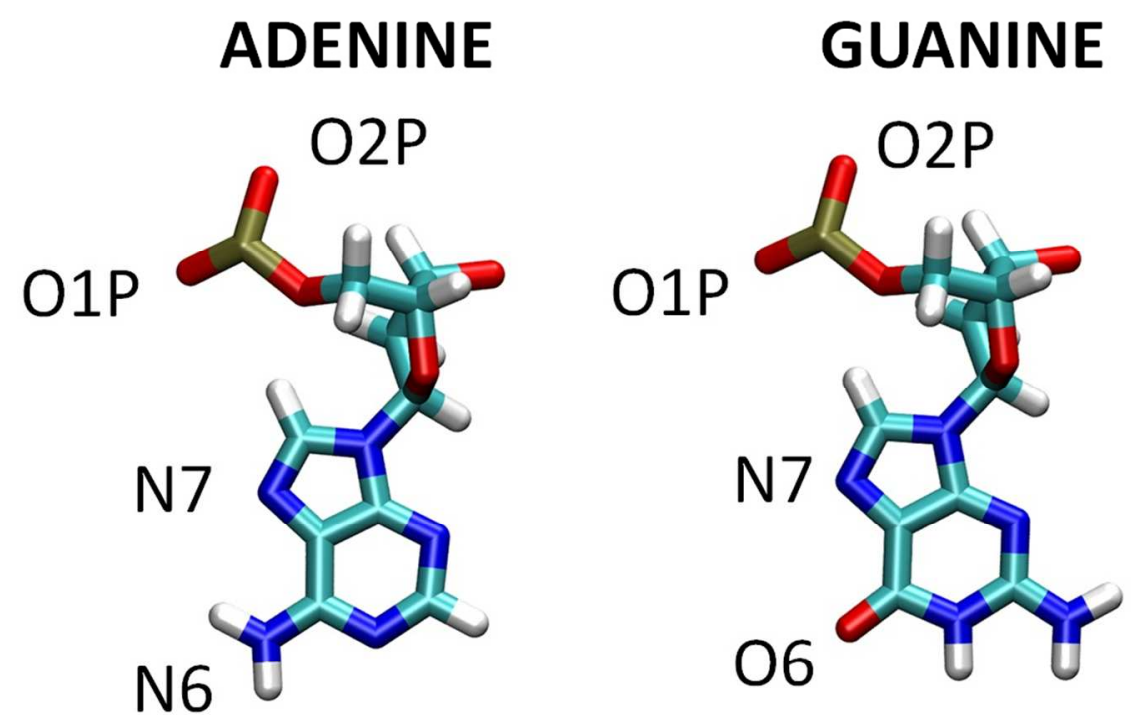

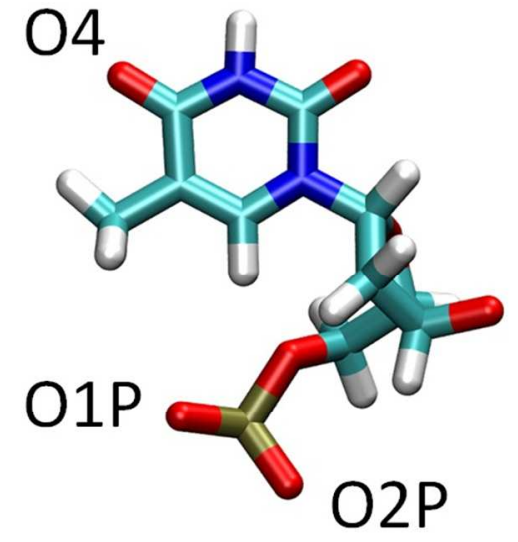

THYMINE

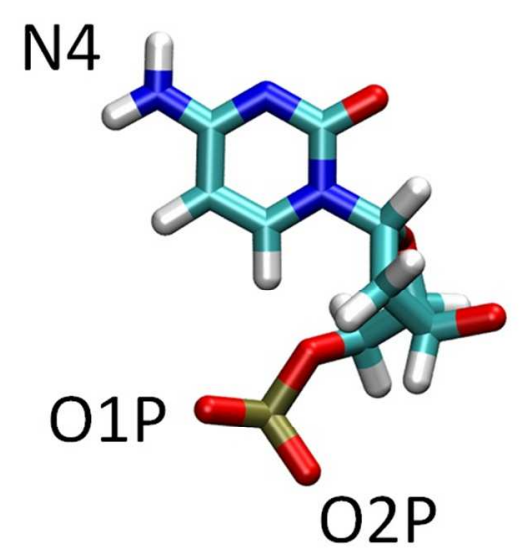

CYTOSINE

Fig. 2 - A•T and C•G in the canonical Watson-Crick DNA base pairing; electronegative reference atoms used in the analysis of ion binding in the major groove and in the backbone are labeled.

Figure 2 
a)

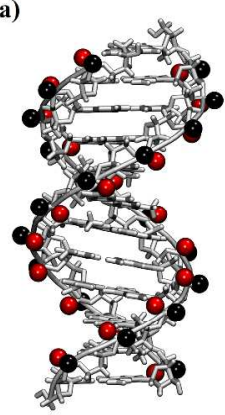

b)

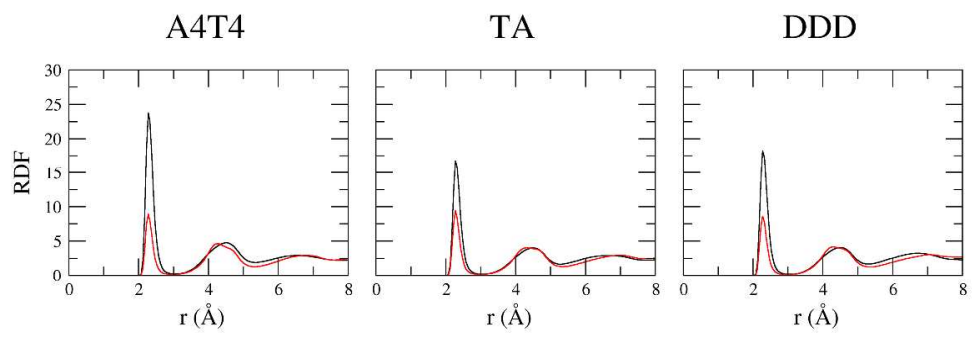

Fig. 3 - (a) The DDD sequence with highlighted oxygen atoms of the phosphates O1P (black spheres) and O2P (red spheres). (b) RDFs of $\mathrm{Na}^{+}$with respect to O1P (black lines) and O2P (red lines) for the three oligomers A4T4, TA and DDD obtained with set 1 parameters.

Figure 3

$404 \times 137 \mathrm{~mm}(300 \times 300$ DPI $)$ 
a)

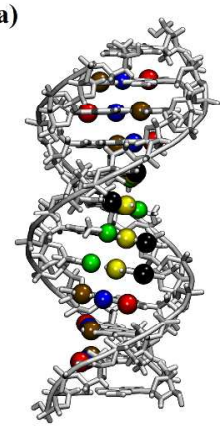

b)

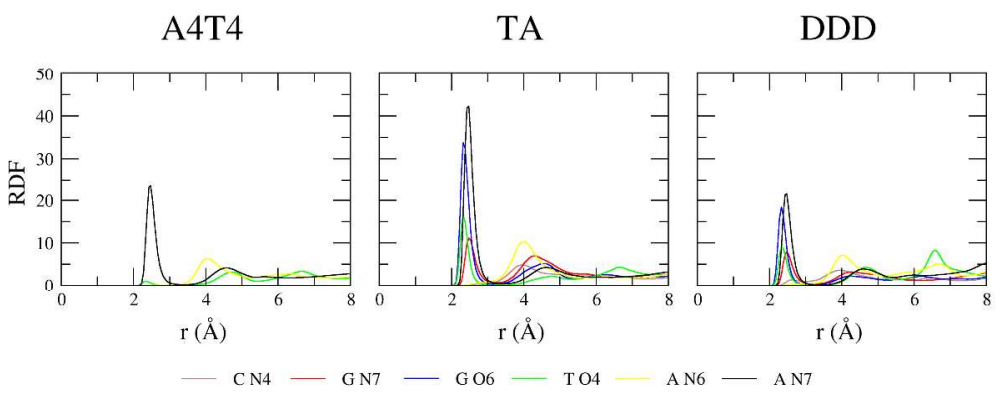

Fig. 4 - (a) The DDD sequence with the highlighted reference atoms of the major groove. (b) RDFs of Na+ with respect to the reference atoms of the major groove for the three oligomers A4T4, TA and DDD obtained with set 1 parameters. Color codes for each atom are shown in the legend.

Figure 4

$390 \times 131 \mathrm{~mm}(300 \times 300 \mathrm{DPI})$ 


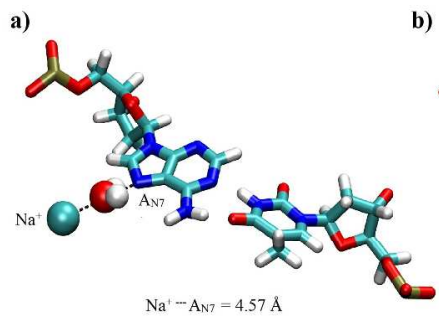

b)
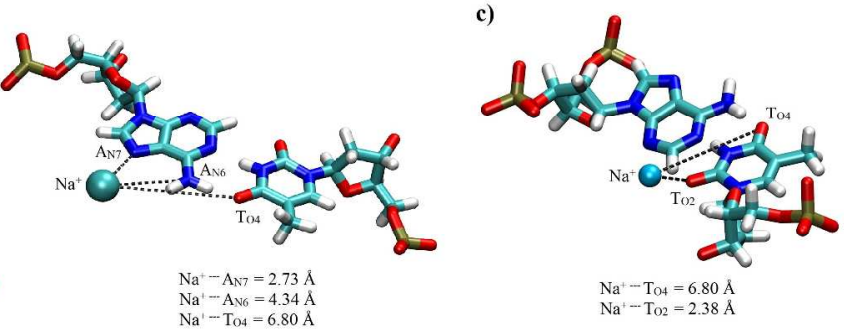

Fig. 5 - a) Representative conformation of a water-mediated contact of a sodium ion with AN7 to explain the peak of the RDF at $\sim 4.5 \AA$. b) Representative conformation of a direct contact of a sodium ion with $A_{N 7}$ to explain the peaks at $\sim 4.5 \AA$ and $\sim 6 \AA$ observed in the peaks of RDF respectively for $A_{N 6}$ and $T_{04}$. C) Representative conformation of a direct contact of a sodium ion with $T_{02}$ to explain the peaks at $\sim 6 \AA$ observed in RDF for $\mathrm{T}_{04}$

Figure 5 

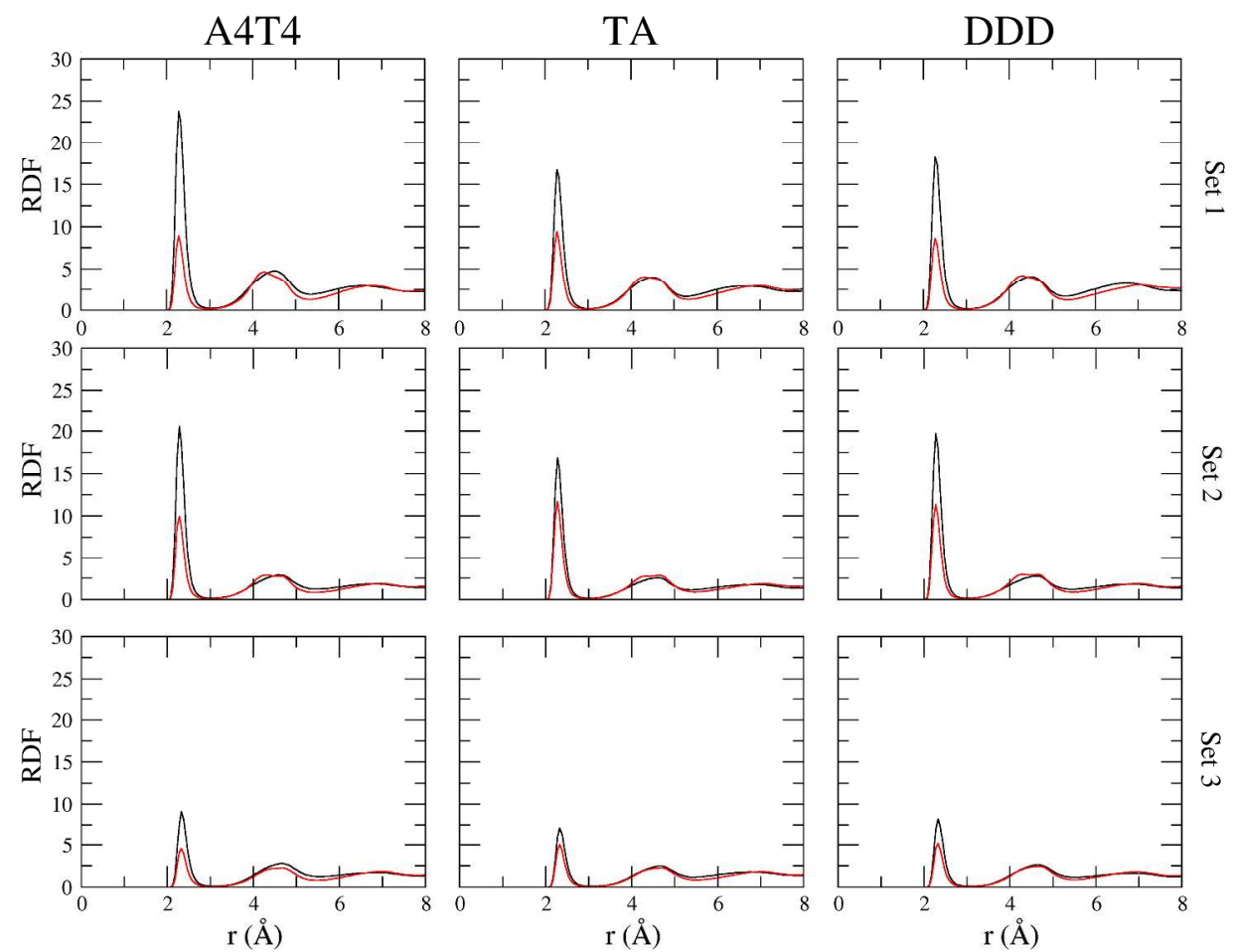

Fig. 6 - RDFs of $\mathrm{Na}^{+}$with respect to O1P (black) and O2P (red) for the three oligomers A4T4, TA and DDD obtained respectively with set 1,2 and 3 parameters.

Figure 6

$326 \times 244 \mathrm{~mm}(300 \times 300 \mathrm{DPI})$ 
A4T4
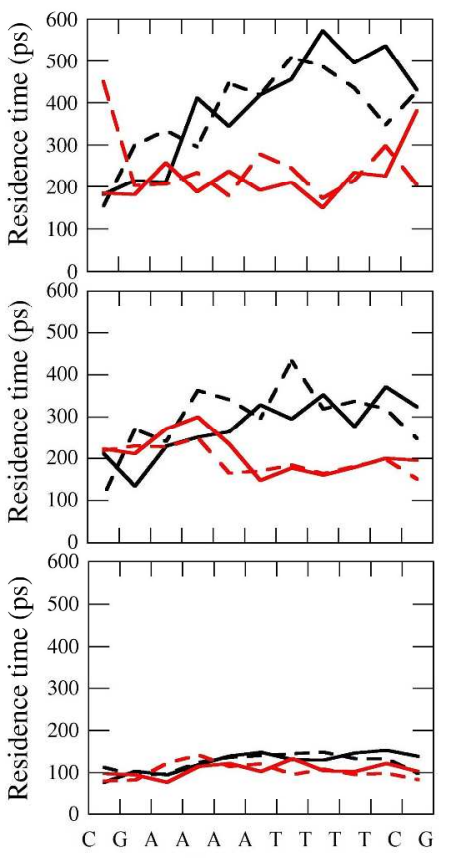

Base sequence
TA
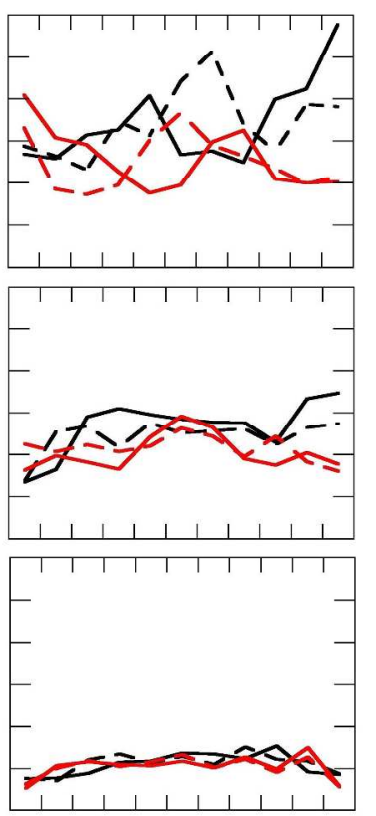

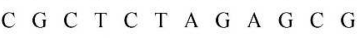
Base sequence
DDD

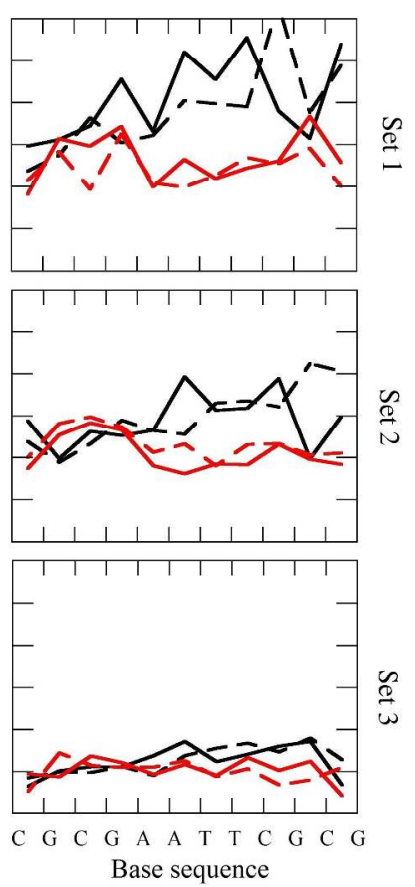

Fig. 7 - Weighted average residence time of $\mathrm{Na}+$ in direct contact with the oxygen atoms of the phosphate groups for the three oligomers A4T4, TA and DDD obtained respectively with set 1,2 and 3 parameters. both filaments are represented in the $5^{\prime} \rightarrow 3^{\prime}$ direction. Color code: black, continuous line = O1P filament 1 ; black dashed line $=01 \mathrm{P}$ filament 2 ; red, continuous line $=02 \mathrm{P}$ filament 1 ; red dashed line $=02 \mathrm{P}$ filament 2.

Figure 7 

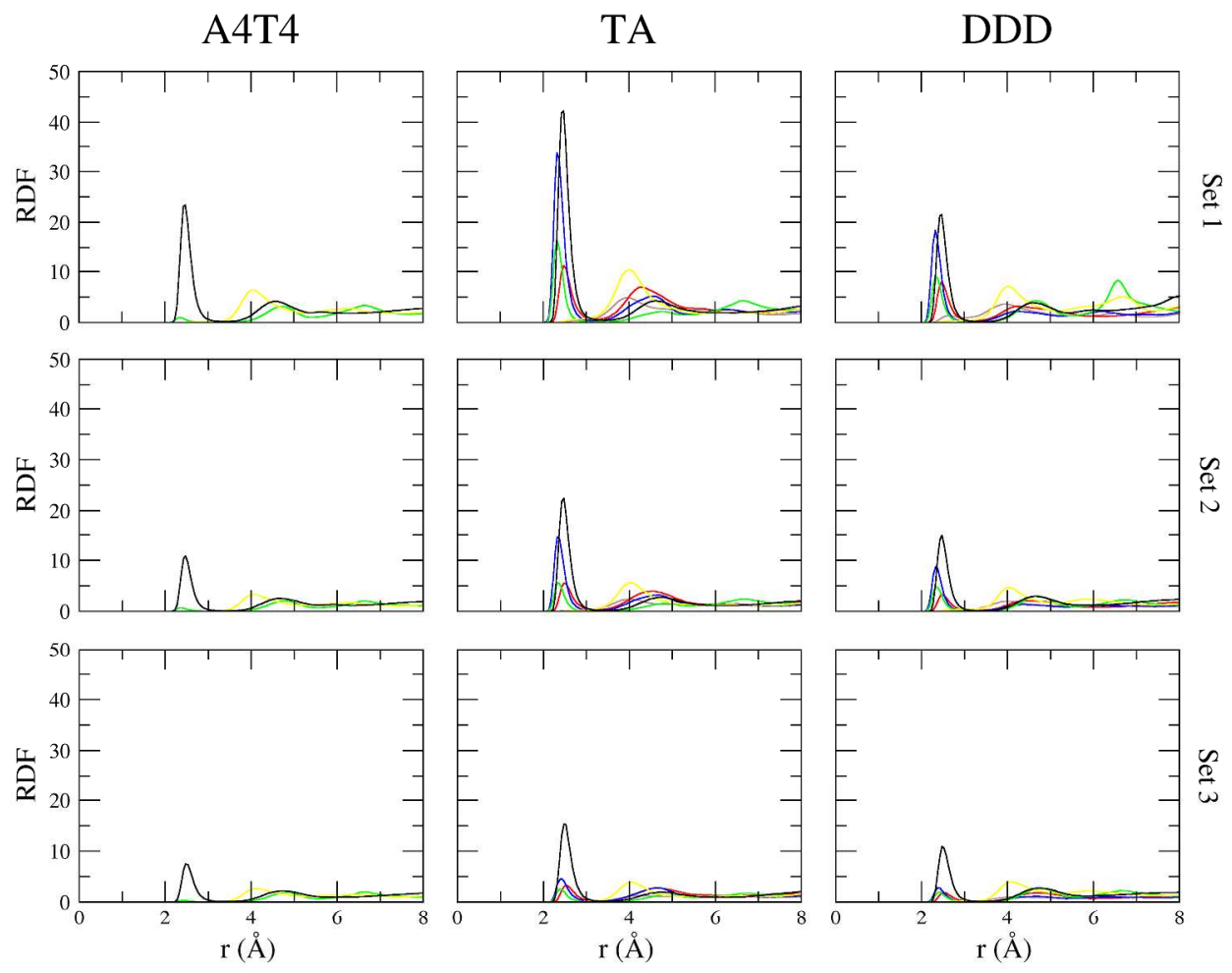

$-\mathrm{CN} 4-\mathrm{GN} 7-\mathrm{GO}-\mathrm{TO} 4-\mathrm{AN6}-\mathrm{AN7}$

Fig. 8 - RDFs of $\mathrm{Na}+$ with respect to reference atoms of the major groove for the three oligomers A4T4, TA and DDD obtained respectively with set 1,2 and 3 parameters. Color codes for each electronegative atom are shown in the legend.

Figure 8

$322 \times 262 \mathrm{~mm}(300 \times 300 \mathrm{DPI})$ 
A4T4
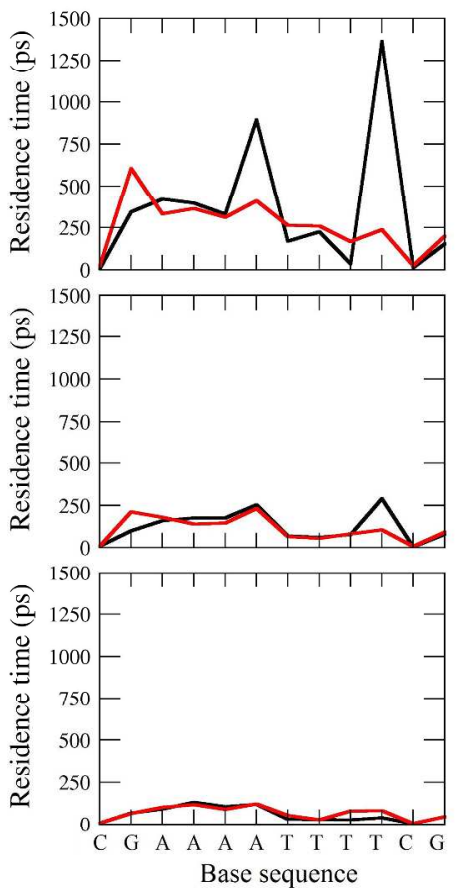

TA
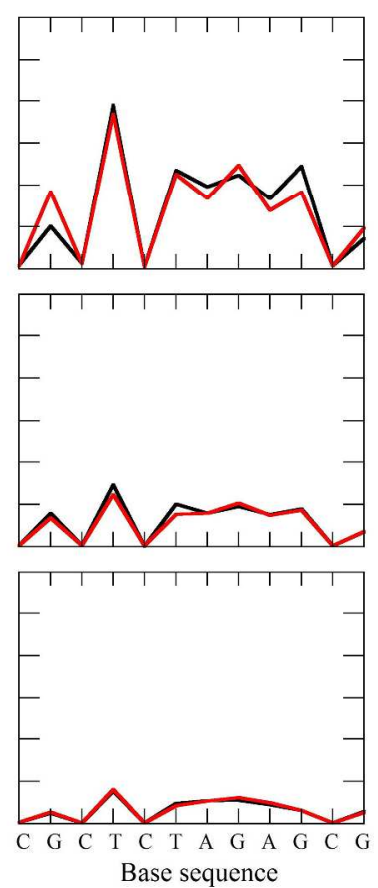

DDD
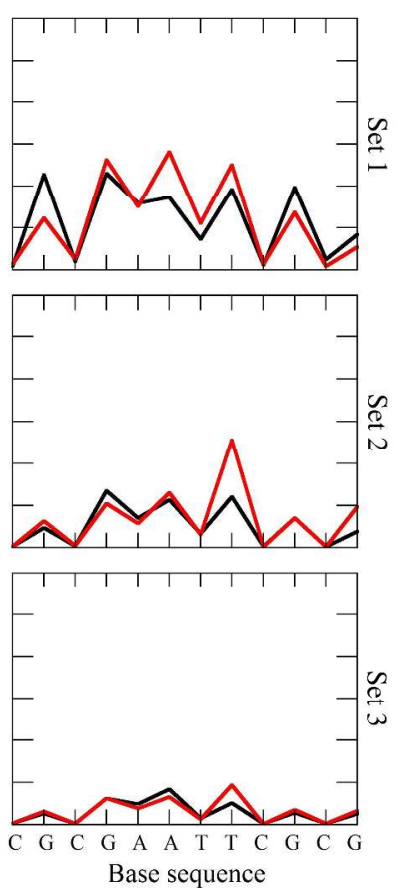

Fig. 9 - Weighted average residence time of $\mathrm{Na}+$ in direct contact with electronegative atoms of the major groove for the three oligomers A4T4, TA and DDD obtained respectively with set 1, 2 and 3 parameters. Colour code: black line $=$ filament 1 ; red line $=$ filament 2 .

Figure 9 